\title{
Correction to: $A P C$ germline variant analysis in the adenomatous polyposis phenotype in Japanese patients
}

\author{
Misato Takao ${ }^{1,2} \cdot$ Tatsuro Yamaguchi $^{1,2,3}$ (1) $\cdot$ Hidetaka Eguchi ${ }^{4} \cdot$ Takeshi Yamada $^{5} \cdot$ Yasushi Okazaki $^{4}$. \\ Naohiro Tomita $^{6} \cdot$ Tadashi Nomizu $^{7} \cdot$ Tomoyuki Momma $^{8} \cdot$ Tetsuji Takayama $^{9} \cdot$ Kohji Tanakaya $^{10} \cdot$ Kiwamu Akagi $^{11}$. \\ Hideyuki Ishida ${ }^{2}$
}

Published online: 20 December 2021

(C) The Author(s) under exclusive licence to Japan Society of Clinical Oncology 2021

\section{Correction to: \\ International Journal of Clinical Oncology \\ (2021) 26:1661-1670 \\ https://doi.org/10.1007/s10147-021-01946-4}

In the original publication, under Study design heading in Methods section, the sentence starting with: "The study was approved by..." should read as:

The study was approved by the Institutional Review Board of Saitama Medical Center at Saitama Medical University (925), the Ethics Committee of Saitama Medical University (747), and the institutional review board at each participating institution.

The original publication has been corrected.

Publisher's Note Springer Nature remains neutral with regard to jurisdictional claims in published maps and institutional affiliations.

The original article can be found online at https://doi.org/10.1007/ s10147-021-01946-4.

Tatsuro Yamaguchi

tatsuro@yamaguchi.email.ne.jp

1 Department of Surgery, Tokyo Metropolitan Cancer and Infectious Diseases Center, Komagome Hospital, 3-18-22, Honkomagome, Bunkyo-ku, Tokyo 113-8677, Japan

2 Department of Digestive Tract and General Surgery, Saitama Medical Center, Saitama Medical University, Saitama, Japan

3 Department of Clinical Genetics, Tokyo Metropolitan Cancer and Infectious Diseases Center, Komagome Hospital, Tokyo, Japan

4 Diagnostics and Therapeutics of Intractable Diseases and Intractable Disease Research Center, Juntendo University Graduate School of Medicine, Tokyo, Japan

5 Department of Gastrointestinal and Hepato-Biliary-Pancreatic Surgery, Nippon Medical School, Tokyo, Japan
6 Division of Lower GI Surgery, Department of Surgery, Hyogo College of Medicine, Nishinomiya, Japan

7 Department of Surgery, Hoshi General Hospital, Koriyama, Japan

8 Department of Gastrointestinal Tract Surgery, Fukushima Medical University, Fukushima, Japan

9 Department of Gastroenterology and Oncology, Institute of Biomedical Sciences, Tokushima University Graduate School, Tokushima, Japan

10 Department of Surgery, Iwakuni Clinical Center, Iwakuni, Japan

11 Division of Molecular Diagnosis and Cancer Prevention, Saitama Cancer Center, Saitama, Japan 